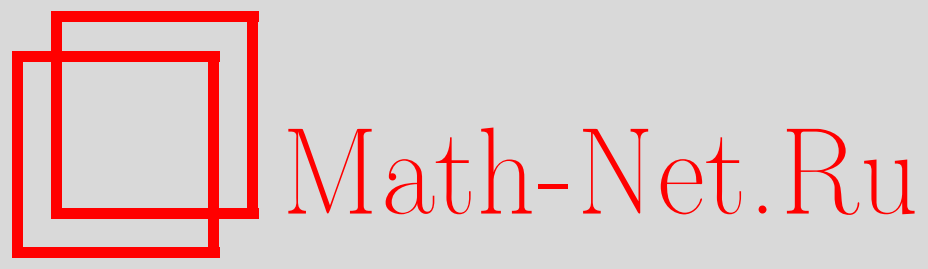

А. Ширинкалам, А. Пураббас, М. Амини, Модульные когомологии и когомологии Хохшильда некоторых полугрупповых алгебр, Функи. анализ и его прил., 2015, том 49, выпуск 4, 90-94

DOI: https://doi.org/10.4213/faa3218

Использование Общероссийского математического портала MathNet.Ru подразумевает, что вы прочитали и согласны с пользовательским соглашением

http://www.mathnet.ru/rus/agreement

Параметры загрузки:

IP : 54.205 .225 .156

26 апреля 2023 г., 14:25:09

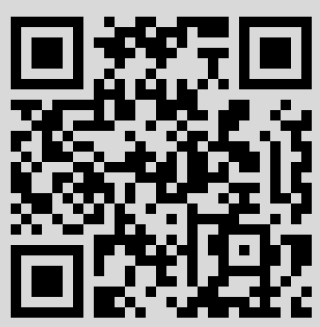


$g \in O(n, \mathbb{R})$ - некоторый фиксированный элемент, коммутирующий с элементами группы $Г$. Рассмотрим в $\mathbb{R}^{n}$ нелокальный оператор типа Каллиаса ${ }^{1)}$, представляющий собой скрещенное произведение вида $D=\not \partial \# a(r) T_{g}$, где $\not \partial-$ оператор Дирака, $a(r)$ - гладкая функция от $r=\sqrt{x_{1}^{2}+\cdots+x_{n}^{2}}$, равная в окрестности бесконечности ненулевой постоянной, а $T_{g}$ - изоморфизм расслоений над отображением $g: \mathbb{R}^{n} \rightarrow \mathbb{R}^{n}$, коммутирующий с действием группы $\Gamma$. Неограниченный оператор $D$ фредгольмов в $L^{2}$, почти коммутирует с действием алгебры $A$ и, следовательно, порождает элемент $[D] \in K^{*}(A)$. Возьмем два экземпляра пространства $\mathbb{R}^{n}$ с оператором $D$ и проведем следующую процедуру разрезания и склеивания: разрежем каждое пространство $\mathbb{R}^{n}$ по сфере $S^{n-1}$ и склеим вырезанные шары по границе в сферу $S^{n}$, а оставшиеся некомпактные части в цилиндр $S^{n-1} \times \mathbb{R}$. Алгебра $A$ естественным образом действует как на шаpe, так и на цилиндре, а из двух копий оператора $D$ получается оператор на сфере $S^{n}$ - дубль оператора $D$, который мы обозначим через $\mathscr{D}$, и оператор $\mathscr{D}_{0}$ на цилиндре. Применение теоремы дает $\tau^{*}[D]+[D]=[\mathscr{D}]+\left[\mathscr{D}_{0}\right]$ в $K^{*}(A)$, где $\tau^{*}: K^{*}(A) \rightarrow K^{*}(A)$ - инволюция, индуцированная отображением $\tau: x \mapsto x / r^{2}$ одноточечной компактификации пространства $\mathbb{R}^{n}$ в себя.

Благодарности. Автор признателен А. Ю. Савину и Б. Ю. Стернину за полезные обсуждения и рецензенту за ценные замечания.

\title{
ЛитеРАтУРА
}

[1] P. B. Gilkey, Invariance Theory, the Heat Equation, and the Atiyah-Singer Index Theorem, CRC Press, Boca Raton, FL, 1995. [2] M. Gromov, H. B. Lawson Jr., Publ. Math. IHES, 58:1 (1983), 295-408. [3] В. Е. Назайкинский, Б. Ю. Стернин, Функц. анализ и его прил., 35:2 (2001), 37-52. [4] V. Nazaikinskii, A. Savin, B.-W. Schulze, B. Sternin, Elliptic Theory on Singular Manifolds, Chapman \& Hall/CRC, Boca Raton, FL, 2005. [5] U. Bunke, Math. Ann., 303:2 (1995), 241-279. [6] N. Higson, J. Roe, Analytic K-Homology, Oxford University Press, Oxford, 2000.

Институт проблем механики им. А. Ю. Ишлинского РАН, Москва Поступило в редакцию Московский физико-технический институт e-mail: nazaikinskii@yandex.ru

\section{Модульные когомологии и когомологии Хохшильда некоторых полугрупповых алгебр}

\author{
(c) 2015. А. Ширинкалам, А. ПураББас, М. Амини
}

1. Введение. Данная статья представляет собой первую попытку систематического изучения взаимосвязи классических и модульных когомологий. Показано, что при определенных условиях, выполненных для некоторого класса примеров, эти когомологии с подходящим образом выбранными коэффициентами совпадают. В частности, это позволяет избавиться от условия коммутативности

1) О классических операторах типа Каллиаса см., например, в [5]. 
в некоторых результатах из [5] и [6] в случае, когда действие предполагается тривиальным слева.

Пусть $\mathscr{L}^{n}(X, Y)$ (соответственно $\left.\mathscr{L}_{\mathfrak{A}}^{n}(X, Y)\right)$ - пространство всех ограниченных $n$-линейных (соответственно модульных) отображений из $X$ в $Y$. Через $X^{(n)}$ обозначим $n$-е сопряженное пространство банахова пространства $X$. Пусть $\mathscr{A}$ и $\mathfrak{A}$ - банаховы алгебры, и пусть $\mathscr{A}$ - банахов $\mathfrak{A}$-бимодуль. Говорят, что действие алгебры $\mathfrak{A}$ на $\mathscr{A}$ совместимо, если для любых элементов $\alpha \in \mathfrak{A}$ и $a, b \in \mathscr{A}$ справедливы равенства $\alpha \cdot(a b)=(\alpha \cdot a) b,(a b) \cdot \alpha=a(b \cdot \alpha)$. Всюду в ра-

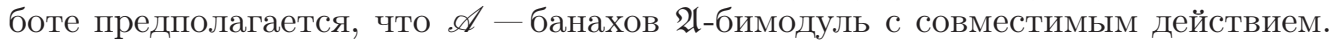
Пусть $X$ является банаховым $\mathscr{A}$-бимодулем и банаховым $\mathfrak{A}$-бимодулем, таким, что для любых элементов $\alpha \in \mathfrak{A}, a \in \mathscr{A}$ и $x \in X$ выполнены соотношения $\alpha \cdot(a \cdot x)=(\alpha \cdot a) \cdot x, a \cdot(\alpha \cdot x)=(a \cdot \alpha) \cdot x$ и $(\alpha \cdot x) \cdot a=\alpha \cdot(x \cdot a)$ и что такие же соотношения выполнены для правых и двусторонних действий. Тогда говорят, что $X$ - банахов $\mathscr{A}$-A-бимодуль. Если, сверх того, $\alpha \cdot x=x \cdot \alpha$ для любых $\alpha \in \mathfrak{A}$ и $x \in X$, то $X$ называется коммутативным банаховым $\mathscr{A}$-А-бимодулем. В этом случае пространство $X^{*}$, сопряженное к пространству $X$, также является коммутативным банаховым $\mathscr{A}$-A-бимодулем с каноническим действием.

Пусть $I$ - замкнутый идеал в проективном тензорном произведении $\mathscr{A} \widehat{\otimes} \mathscr{A}$, порожденный элементами вида $a \cdot \alpha \otimes b-a \otimes \alpha \cdot b$, где $\alpha \in \mathfrak{A}$ и $a, b \in \mathscr{A}$. Тогда модульное проективное тензорное произведение $\mathscr{A} \widehat{\otimes}_{\mathfrak{A}} \mathscr{A}$ - это факторалгебра $(\mathscr{A} \widehat{\otimes} \mathscr{A}) / I[4]$. Пусть $J$ - замкнутый идеал в $\mathscr{A}$, порожденный множеством $\pi(I)$, где $\pi: \mathscr{A} \widehat{\otimes} \mathscr{A} \rightarrow \mathscr{A}$ - отображение умножения.

Пусть $X$ - банахов $\mathscr{A}$-A-бимодуль. Ограниченное линейное отображение $D$ : $\mathscr{A} \rightarrow X$ называется $\mathfrak{A}$-модульным дифференцированием, если оно является дифференцированием и отображением $\mathfrak{A}$-модулей. Если $X-$ коммутативный $\mathscr{A}$-А-бимодуль, то для любого $x \in X$ отображение $\operatorname{ad}_{x}: \mathscr{A} \rightarrow X$, заданное формулой $\operatorname{ad}_{x}(a):=a \cdot x-x \cdot a$, является модульным дифференцированием и называется внутренним модульным дифференцированием. Пространство всех $\mathfrak{A}$-модульных дифференцирований, действующих из $\mathscr{A}$ в $X$, обозначается через $\mathscr{Z}_{\mathfrak{A}}^{1}(\mathscr{A}, X)$, а подпространство внутренних модульных дифференцирований обозначается через $\mathscr{B}_{\mathfrak{A}}^{1}(\mathscr{A}, X)$. Первая группа $\mathfrak{A}$-модульных когомологий алгебры $\mathscr{A}$ с коэффициентами в $X$ определяется как полунормированное факторпространство $\mathscr{H}_{\mathfrak{A}}^{1}(\mathscr{A}, X):=\mathscr{Z}_{\mathfrak{A}}^{1}(\mathscr{A}, X) / \mathscr{B}_{\mathfrak{A}}^{1}(\mathscr{A}, X)$. Определение высших групп модульных когомологий можно найти в [6].

\section{2. Взаимосвязь модульных когомологий и когомологий Хохшиль-} да. Пусть $\mathscr{A}, \mathfrak{A}$ и замкнутый идеал $J$ те же, что и в предыдущем разделе, и пусть $X$ - банахов $\mathscr{A}$-А-бимодуль. В данном разделе мы получим достаточные условия для того, чтобы группы когомологий $\mathscr{H}_{\mathfrak{A}}^{1}(\mathscr{A}, X)$ и $\mathscr{H}^{1}(\mathscr{A} / J, X)$ были изоморфны как полунормированные пространства. Будем говорить, что действие алгебры $\mathfrak{A}$ на $X$ тривиально слева, если для любых $\alpha \in \mathfrak{A}$ и $x \in X$ справедливо равенство $\alpha \cdot x=f(\alpha) x$, где $f$ - характер на $\mathfrak{A}$.

Всюду в этом разделе предполагается, что $X$-коммутативный банахов $\mathscr{A}$-A-бимодуль, причем действие алгебры $\mathfrak{A}$ как на $\mathscr{A}$, так и на $X$ тривиально слева при одном и том же характере $f$ на $\mathfrak{A}$. Мы также предполагаем, что банахова алгебра $A / J$ унитальна. В следующем разделе представлены примеры, удовлетворяющие всем этим условиям.

Предложение 1. Банаховы пространства $\mathscr{Z}_{\mathfrak{A}}^{1}(\mathscr{A}, X)$ и $\mathscr{Z}^{1}(\mathscr{A} / J, X)$ изометрически изоморфны. 
Доказательство. Отображение $\rho: \mathscr{Z}_{\mathfrak{A}}^{1}(\mathscr{A}, X) \rightarrow \mathscr{Z}^{1}(\mathscr{A} / J, X)$, определенное для каждого $D \in \mathscr{Z}_{\mathfrak{A}}^{1}(\mathscr{A}, X)$ формулой $\rho(D)(a+J)=D(a)$, является сюръективной изометрией.

Теорема 2. Полунормированные пространства $\mathscr{H}_{\mathfrak{A}}^{1}(\mathscr{A}, X)$ и $\mathscr{H}^{1}(\mathscr{A} / J, X)$ изоморфны.

Доказательство. Изоморфизм $\rho$ из предыдущего предложения отображает внутренние модульные дифференцирования на $\mathscr{A}$ во внутренние дифференцирования на $\mathscr{A} / J$ и, следовательно, индуцирует сюръективное отображение

$$
\Phi: \mathscr{Z}_{\mathfrak{A}}^{1}(\mathscr{A}, X) / \mathscr{B}_{\mathfrak{A}}^{1}(\mathscr{A}, X) \rightarrow \mathscr{Z}^{1}(\mathscr{A} / J, X) / \mathscr{B}^{1}(\mathscr{A} / J, X),
$$

задаваемое формулой $\Phi\left(D+\mathscr{B}_{\mathfrak{A}}^{1}(\mathscr{A}, X)\right):=\rho(D)+\mathscr{B}^{1}(\mathscr{A} / J, X)$. Простая проверка показывает, что $\Phi$ - изоморфизм полунормированных пространств.

В следующей лемме при некоторых условиях отождествляются банаховы пространства $\mathscr{A} \widehat{\otimes}_{\mathfrak{A}} X$ и $\mathscr{A} / J \widehat{\otimes} X$.

Напомним, что $J$ - замкнутый идеал в $\mathscr{A}$, совпадающий с замкнутой линейной оболочкой множества элементов вида $(a \cdot \alpha) b-a(\alpha \cdot b)$, где $\alpha \in \mathfrak{A}$ и $a, b \in \mathscr{A}$. Пусть $J_{0}$ - замкнутая линейная оболочка множества элементов вида $a \cdot \alpha-f(\alpha) a$, где $a \in \mathscr{A}$ и $\alpha \in \mathfrak{A}$. Так как предполагается, что алгебра $\mathscr{A} / J$ унитальна, то $J_{0} \subseteq J$. На практике в наиболее интересных примерах (см. следующий раздел) оказывается, что $J=J_{0}$.

Лемма 3. Пусть в предыдущих обозначениях $J=J_{0}$, а $X$ есть $\mathfrak{L}_{1}$-пространство $^{1)}$. Тогда банаховы пространства $\mathscr{A} / J \widehat{\otimes} X$ и $\mathscr{A} \widehat{\otimes}_{\mathfrak{A}} X$ изометрически изоморфны.

Кратко объясним доказательство леммы. Из определения проективного тензорного произведения модулей следует, что $\mathscr{A} \widehat{\otimes} \mathfrak{A} X \simeq \mathscr{A} \widehat{\otimes} X / I_{0}$, где $I_{0}-$ это замкнутая линейная оболочка множества $\{a \cdot \alpha \otimes x-a \otimes \alpha \cdot x, a \in \mathscr{A}, \alpha \in \mathfrak{A}$, $x \in X\}$. Рассмотрим естественную проекцию $q: \mathscr{A} \rightarrow \mathscr{A} / J$. Тогда отображение $\varphi:=q \otimes \mathrm{id}: \mathscr{A} \widehat{\otimes} X \rightarrow \mathscr{A} / J \widehat{\otimes} X$, заданное формулой $\varphi(a \otimes x):=(a+J) \otimes x$ для любых $a \in \mathscr{A}$ и $x \in X$, является естественной проекцией на образ, так что в силу предложения 2.5 из [10] получаем изометрический изоморфизм $\mathscr{A} \widehat{\otimes} X / \operatorname{ker} \varphi \simeq$ $\mathscr{A} / J \widehat{\otimes} X$. Теперь из теоремы 2.20 в [10] следует, что $\operatorname{ker} \varphi=J \widehat{\otimes} X$. Наконец, можно показать, что $J \widehat{\otimes} X=I_{0}$.

Предложение 4 (редукция размерности). Пусть $\mathscr{A} / J$ является $\mathfrak{L}_{1}$-пространством. В предположениях леммы 3 справедливы следующие утверждения:

(i) $\mathscr{L}_{\mathfrak{A}}^{k}\left(\mathscr{A}, X^{*}\right) \simeq(\underbrace{\mathscr{A} \widehat{\otimes}_{\mathfrak{A}} \cdots \widehat{\otimes}_{\mathfrak{A} \mathscr{A}}}_{k \text { раз }} \widehat{\otimes}_{\mathfrak{A}} X)^{*}-$ коммутативныи банахов $\mathscr{A}-\mathfrak{A}-$ бимодуль для каждого $k \in \mathbb{N}$;

(ii) имеет место изоморфизм $\mathscr{H}_{\mathfrak{A}}^{n+k}(\mathscr{A}, X) \simeq \mathscr{H}_{\mathfrak{A}}^{n}\left(\mathscr{A}, \mathscr{L}_{\mathfrak{A}}^{k}(\mathscr{A}, X)\right)$ полунормированных пространств для любых $k, n \in \mathbb{N}$.

Изометрический изоморфизм в п. (i) следует из упражнения 5.3.1 в [9]. Аналогичное рассуждение из доказательства теоремы 2.4.6 в [9] с заменой коцепей на модульные коцепи доказывает утверждение п. (ii).

1) За определением $\mathfrak{L}_{1}$-пространства мы отсылаем читателя к [10]. Примерами таких пространств являются пространство $L^{1}(\mu)$ для заданной меры $\mu$ и пространство $C(K)^{*}$ для заданного компактного пространства $K$. 
Следствие 5. При тех же предположениях, что и выше, пространства $\mathscr{H}_{\mathfrak{A}}^{k}\left(\mathscr{A}, X^{*}\right)$ и $\mathscr{H}^{k}\left(\mathscr{A} / J, X^{*}\right)$ изометрически изоморфны.

Доказательство. В силу предложения 4 и теоремы 2 имеем

$$
\begin{aligned}
\mathscr{H}_{\mathfrak{A}}^{k}\left(\mathscr{A}, X^{*}\right) \simeq \mathscr{H}^{1}\left(\mathscr{A} / J, \mathscr{L}_{\mathfrak{A}}^{k-1}\left(\mathscr{A}, X^{*}\right)\right) \\
\simeq \mathscr{H}^{1}\left(\mathscr{A} / J, \mathscr{L}^{k-1}\left(\mathscr{A} / J, X^{*}\right)\right) \simeq \mathscr{H}^{k}\left(\mathscr{A} / J, X^{*}\right) .
\end{aligned}
$$

Заметим, что так как п. (i) предложения 4 выполнен только для сопряженных модулей $X^{*}$, то и следствие 5 верно только для них.

3. Приложения к полугрупповым алгебрам. Пусть $S$ - инверсная полугруппа с множеством $E$ идемпотентов. Пусть $\ell^{1}(E)$ действует на $\ell^{1}(S)$ умножением справа и тривиально слева, т. е. $\delta_{e} \cdot \delta_{s}=\delta_{s}, \delta_{s} \cdot \delta_{e}=\delta_{s e}=\delta_{s} * \delta_{e}(e \in E$, $s \in S$ ), где $\delta_{s}$ - единичная масса в точке $s$. Здесь замкнутый идеал $J$ (см. разд. 1) - это замкнутая линейная оболочка множества $\left\{\delta_{\text {set }}-\delta_{s t}: s, t \in S\right.$, $e \in E\}$. Рассмотрим на множестве $S$ отношение эквивалентности, определенное следующим образом:

$$
s \sim t \Longleftrightarrow \delta_{s}-\delta_{t} \in J \quad(s, t \in S)
$$

Из рассуждений, предшествующих теореме 2.4 в [1], следует, что $S / \sim-$ дискретная группа. В этом случае в силу доказательства теоремы 3.3 из [8] можно заметить, что $\ell^{1}(S) / J \simeq \ell^{1}(S / \sim)$ как (коммутативные) $\ell^{1}(E)$-бимодули. Дискретная группа $S / \sim$ совпадает с максимальным гомоморфным групповым образом $G_{S}$ полугруппы $S$.

Насрабади и второй автор [6] показали, что для коммутативной инверсной полугруппы $S$ с множеством $E$ идемпотентов группа когомологий $\mathscr{H}_{\ell^{1}(E)}^{1}\left(\ell^{1}(S)\right.$, $\left.\ell^{1}(S)^{(2 n+1)}\right)$ тривиальна для любого $n \in \mathbb{N}$. В общем случае, когда $S$ некоммутативна, а $\ell^{1}(E)$ действует на $\ell^{1}(S)$ умножением справа и тривиально слева, $\ell^{1}(S)$ не обязательно коммутативна как банахов $\ell^{1}(E)$-бимодуль. Тем не менее, если рассмотреть $\ell^{1}(S) / J$ как банахов $\ell^{1}(E)$-бимодуль, то при сделанных выше предположениях $\ell^{1}(S) / J$ оказывается коммутативным $\ell^{1}(S)-\ell^{1}(E)$-бимодулем. Мы покажем, что группа когомологий $\mathscr{H}_{\ell^{1}(E)}^{1}\left(\ell^{1}(S),\left(\ell^{1}(S) / J\right)^{(2 n+1)}\right)$ тривиальна для каждого $n \in \mathbb{N}$.

Теорема 6. Пусть $S-$ инверсная полугруппа с множеством Е идемпотентов. Путь $\ell^{1}(E)$ действует на $\ell^{1}(S)$ умножсением справа и тривиально слева. Тогда $\mathscr{H}_{\ell^{1}(E)}^{1}\left(\ell^{1}(S), \ell^{1}\left(G_{S}\right)^{(2 n+1)}\right)=0$ для любого $n \in \mathbb{N} \cup\{0\}$.

Доказательство. Для каждого $s \in S$, полагая $e=s^{*} s$, получаем $\delta_{s}-\delta_{s e} \in J$, так что $\ell^{1}\left(G_{S}\right)=\ell^{1}(S) / J-$ коммутативный банахов $\ell^{1}(S)-\ell^{1}(E)$-бимодуль. По теореме 2.2 из [7] и теореме 2 имеем

$$
\begin{aligned}
\mathscr{H}_{\ell^{1}(E)}^{1}\left(\ell^{1}(S), \ell^{1}\left(G_{S}\right)^{(2 n+1)}\right) \simeq \mathscr{H}^{1}\left(\ell^{1}(S) / J\right. & \left.\left(\ell^{1}(S) / J\right)^{(2 n+1)}\right) \\
& \simeq \mathscr{H}^{1}\left(\ell^{1}\left(G_{S}\right),\left(\ell^{1}\left(G_{S}\right)\right)^{(2 n+1)}\right)=0 .
\end{aligned}
$$

Далее мы обобщаем следствие 3.5 из [6] с клиффордовых полугрупп на произвольные инверсные полугруппы.

Теорема 7. Пусть $S$ - инверсная полугруппа с множеством $E$ идемпотентов. Пусть $\ell^{1}(E)$ действует на $\ell^{1}(S)$ умножсением справа и тривиально 
слева. Тогда для любого $n \in \mathbb{N} \cup\{0\}$ группа гомологий $\mathscr{H}_{\ell^{1}(E)}^{2}\left(\ell^{1}(S), \ell^{1}\left(G_{S}\right)^{(2 n+1)}\right)$ является банаховым пространством.

Доказательство. Заметим, что для любой локально компактной группы $G$ и любого $n \in \mathbb{N}$ существует компактное хаусдорфово пространство $K_{n}$, такое, что $L^{1}(G)^{(2 n)} \simeq M\left(K_{n}\right)$ является $\mathfrak{L}_{1}$-пространством. Поскольку $\delta_{\text {set }}-\delta_{s t}=$ $\delta_{s e t t^{*} t}-\delta_{s t}=\delta_{s t t^{*} e t}-\delta_{s t}$ и $t^{*} e t-$ идемпотент, скажем, $e^{\prime}$, получаем, что $\delta_{s e t}-$ $\delta_{s t}=\delta_{r e^{\prime}}-\delta_{r}$ при $r=s t$. Таким образом, элементы вида $\delta_{r} \cdot \delta_{e^{\prime}}-f\left(e^{\prime}\right) \delta_{r}$ порождают $J$, где $f$-аугментационный характер на $\ell^{1}(E)$. Теперь при $n=0$ получаем, что $\ell^{1}(S) / J \simeq \ell^{1}\left(G_{S}\right)$ - унитальная банахова алгебра и $\mathfrak{L}_{1}$-пространство. Также и $\ell^{1}(S) / J \widehat{\otimes} \ell^{1}\left(G_{S}\right) \simeq \ell^{1}\left(G_{S} \times G_{S}\right)$ является $\mathfrak{L}_{1}$-пространством. Поэтому, согласно следствию 5 ,

$$
\mathscr{H}_{\ell^{1}(E)}^{2}\left(\ell^{1}(S), \ell^{\infty}\left(G_{S}\right)\right) \simeq \mathscr{H}^{2}\left(\ell^{1}(S) / J, \ell^{\infty}\left(G_{S}\right)\right) \simeq \mathscr{H}^{2}\left(\ell^{1}\left(G_{S}\right), \ell^{\infty}\left(G_{S}\right)\right) .
$$

В силу теоремы 3.3 из [7] последнее пространство банахово. При $n \geqslant 1$, поскольку $\ell^{1}\left(G_{S}\right)^{(2 n)}$ есть $\mathfrak{L}_{1}$-пространство, получаем, что $\left.\mathscr{H}_{\ell^{1}(E)}^{2}\left(\ell^{1}(S), \ell^{1}\left(G_{S}\right)\right)^{(2 n+1)}\right)$ $\simeq \mathscr{H}^{2}\left(\ell^{1}\left(G_{S}\right),\left(\ell^{1}\left(G_{S}\right)\right)^{(2 n+1)}\right)$ в силу следствия 5. Снова по теореме 3.3 из [7] последнее пространство банахово.

\section{ЛиТЕРАТУРА}

[1] M. Amini, A. Bodaghi, D. Ebrahimi Bagha, Semigroup Forum, 80:2 (2010), 302312. [2] H. G. Dales, Banach Algebras and Automatic Continuity, Clarendon Press, Oxford, 2000. [3] H. G. Dales, A. T.-M. Lau, D. Strauss, Dissertationes Math. (Rozprawy Mat.), 481 (2011), 1-121. [4] А. Я. Хелемский, Гомология в банаховых и топологических алгебрах, Изд-во МГУ, М., 1986. [5] E. Nasrabadi, A. Pourabbas, Semigroup Forum, 81:2 (2010), 269-276. [6] E. Nasrabadi, A. Pourabbas, Bull. Iranian Math. Soc., 37:4 (2011), 157-168. [7] A. Pourabbas, Proc. Amer. Math. Soc., 132:2 (2004), 1403-1410. [8] R. Rezavand, M. Amini, M. H. Sattari, and D. Ebrahimi Bagha, Semigroup Forum, 77:2 (2008), 300-305. [9] V. Runde, Lectures on Amenability, Lecture Notes in Mathematics, vol. 1774, Springer-Verlag, Berlin, 2002. [10] R. A. Ryan, Introduction to Tensor Products of Banach Spaces, Springer-Verlag, London, 2002.

Faculty of Mathematics and Computer Science, Amirkabir University of Technology, Tehran, Iran e-mail: shirinkalam_a@aut.ac.ir

Faculty of Mathematics and Computer Science, Amirkabir University of Technology, Tehran, Iran e-mail: arpabbas@aut.ac.ir

Department of Mathematics, Tarbiat Modares University

School of Mathematics, Institute for Research in Fundamental Sciences (IPM), Tehran, Iran e-mails: mamini@modares.ac.ir, mamini@ipm.ir
Поступила в редакцию 26 сентября 2014 г. Переработанный вариант 1 марта 2015 г. 